\title{
Factors that impact pharmacokinetic measurements of antibody therapeutics: what is your PK assay telling you?
}

\author{
Saloumeh Kadkhodayan Fischer ${ }^{*} 1$ \\ 1'Assay Development \& Technology, Genentech Inc., 1 DNA Way, South San Francisco, CA 94080, USA \\ * Author for correspondence: Tel.: +1 650225 4153; Fax: +1 650 2251998; sallyk@gene.com
}
'6“... assay reagents, format and conditions can significantly impact the PK parameters/profiles for an antibody therapeutic depending on what drug species is being detected.""

First draft submitted: 21 July 2017; Accepted for publication: 15 August 2017; Published online: 26 October 2017

Keywords: free drug $\bullet$ LBA $\bullet$ ligand binding assay $\bullet$ pharmacokinetics $\bullet$ PK • total drug

"... assay reagents, format and conditions can significantly impact the PK parameters/profiles for an antibody therapeutic depending on what drug species is being detected."

Pharmacokinetic (PK) measurements are critical in all phases of antibody therapeutic (AbT) development, starting from target/molecule selection during the discovery stage, PK projections from animal to human to inform doses for early clinical studies, to safety/efficacy evaluation during proof-of-concept and confirmatory clinical studies (Phase II/III). Due to the bivalent nature of antibodies, in presence of target they can exist in different forms including fully bound, partially bound or unbound forms. Total drug ( $\left.\mathrm{AbT}_{\text {total }}\right)$ refers to all forms of the drug present, as opposed to free drug $\left(\mathrm{AbT}_{\text {free }}\right)$, which refers to both partially bound form where one of the antibody arms is complexed with a target and/or completely unbound drug that has both antibody arms available for binding. In the majority of cases where the circulating target levels are low or when AbT concentrations are maintained at higher levels than their therapeutic target in order to produce a pharmacologic effect, the 'total' and 'free' drug levels are practically equivalent [1]. However, in the presence of high levels of circulating target, different complexes can coexist and 'total' and 'free' drug may differ significantly [2,3]. In addition to circulating targets, anti-drug antibodies can bind to the drug and impact PK measurements. Therefore it is important to understand which form of drug is being measured in the assay in order to properly interpret the drug concentrations and characterize the PK of therapeutic antibodies.

\section{Free versus total drug: which is most relevant?}

Typically, PK scientists are interested in $\mathrm{AbT}_{\text {free }}$ as it is the pharmacological active form of the drug and used to project PK from animal to human, calculate safety margins, determine PK/PD relationships and inform dose selection. Toxicologists on the other hand, may be more interested in total drug exposure $\left(\mathrm{AbT}_{\text {total }}\right)$ and potential safety concerns. Information from $\mathrm{AbT}_{\text {total }}$ may also be required to gain an understanding of the dynamics of the drug/target interaction and drug clearance. There has been a lot of interest and discussion on this topic. There is growing interest in measurement of $\mathrm{AbT}_{\text {free }}$ or pharmacologically active forms of the drug, as these levels would reflect the impact of drug exposure by soluble ligand and neutralizing antibodies and are thought to better correlate with efficacy. Still, the debate on this topic continues as there are different opinions on the subject [4].

In October 2008, the ligand-binding assay (LBA) bioanalytical focus group (LBABFG) from the American Association of Pharmaceutical Scientists (AAPS) sponsored a forum to debate the challenges and issues in measuring 'free' and 'total' drugs. These discussions resulted in a review article as well as a consensus-based 'AAPS White Paper' [1,4]. Since then there has been ongoing dialogue, including this year's discussions at the 11th Workshop on Recent Issues in Bioanalysis (WRIB), highlighting the continued interest in this topic. At the 11th WRIB meeting, which included experts from industry and regulatory bodies, agreement was reached that the decision as to what 
form of the drug is appropriate for detection should be based solely on the disease biology and the mode of action of the therapeutic. Therefore, it is important for the bioanalytical scientist and all of the stakeholders interpreting the data (e.g., PK scientist, toxicologist and PK/PD modeler) to understand and discuss:

1. The intended use of the bioanalytical assay data;

2. The biology and rationale for what drug species to measure;

3. The anticipated target concentrations;

4. Technical challenges and practical limitations of the assays and reagent availability.

\section{Bioanalytical considerations}

In the presence of a circulating target, the equilibrium between free and complexed forms of the drug is dynamic. Although in theory an assay could be designed to measure free or total levels of drug, measurement of $\mathrm{AbT}_{\text {free }}$ is technically very challenging. This is due to the fact that the equilibrium of free/complexed drug can be significantly altered by the choice of assay format, reagents and conditions, which can in turn impact the interpretation of the PK of the therapeutic.

\section{Durg + Target $\rightleftharpoons$ Drug : Target}

One factor that can further complicate measurement of $\mathrm{AbT}_{\text {free }}$ is the level of target at baseline versus postdose. It is often difficult to predict the levels of soluble target postdose and therefore the impact on PK parameter estimates for a new biotherapeutic program. Although the baseline levels of target may be low and at times undetectable, these levels can increase significantly postdose. This is due to a decreased clearance rate of the complexed target compared with free target $[5,6]$. The increase in target level due to complex formation can be as much as 100 -fold of baseline levels and depends on the target turnover rate and the affinity of drug for target and drug levels [7]. The presence of these complexes can significantly influence drug/target complex equilibrium, depending on the choice of assay format as well as assay conditions such as incubation time and the extent of sample dilution. The assay conditions can in turn also impact the equilibrium in LBA between the drug and the assay reagents [8]. Due to the limited dynamic range of most assays, almost all samples (except for very low concentrations) have to be diluted in order to bring the drug concentration of the samples into the quantifiable calibration range. Since drug levels during the course of clinical studies vary based on time of sample collection, the dilution scheme could be very different for each sample. Higher dilutions can promote the dissociation of existing drug/target complexes and shift the equilibrium toward free drug, depending on the binding affinity of the antibody for the target. Consequently the levels of free drug measured in an assay may not be representative of post-dose circulating levels in vivo.

Another factor to be considered in free assays is a need for 'ligand-free' matrix for accurate free drug quantification [7-9]. Based on regulatory guidance, matrix effects should be evaluated by comparing calibration standard curves prepared in buffer to that prepared in matrix to demonstrate lack of matrix effect [10]. However, since the intended matrix often contains some levels of target, this can be difficult. In most cases, depleted or surrogate matrix needs to be utilized and may not be completely representative of the original matrix. One concept that is being implemented in free assay validations is the use of free analyte quality control (QC) samples, which are control samples containing a defined amount of free and ligand complexes. Use of these QC samples can enable the evaluation of factors that may impact the drug/target complex equilibrium. In contrast to free assays, measurement of $\mathrm{AbT}_{\text {total }}$ is not sensitive to changes in the drug/ligand equilibrium; therefore it is less prone to impact from assay conditions and implemented methods.

When measuring total drug, different reagents may be used for capture and detection: non-neutralizing anticomplementarity determining region (CDR) monoclonal antibodies, CDR-specific polyclonal antibodies and/or anti-framework antibodies can be used for capture and detection. The most commonly used formats for free drug measurements are ligand/target binding or anti-idiotypic antibody binding assays. These formats require at least one of the antibody drug arms to be available for binding and measure either partially bound or totally free drug, depending on the detection reagent used. For example, if anti-idiotypic antibody or ligand is used for detection, the assay would measure completely free or unbound drug. On the other hand, if non-neutralizing anti-CDR antibodies or generic reagents such as anti-framework antibodies are used, the assay would measure partially bound drug. However, in the absence of ideal reagents, assay conditions can be manipulated to shift the equilibrium between free and complexed drug. The conditions of an LBA can be modified to measure total drug by simply 
increasing the assay incubation time [11]. It is therefore essential to understand the impact of assay conditions on the specific form of the drug that is being measured. This can be accomplished by evaluating drug recovery at different target to drug molar ratios and optimizing the assay conditions to tolerate the expected target levels [11].

\section{Conclusion}

In the presence of high concentrations of circulating target, assay reagents, format and conditions can significantly impact the PK parameters/profiles for an AbT depending on what drug species is being detected. Therefore, understanding the assay as well as the strengths and limitations of the resulting data are crucial for data interpretation and to draw accurate conclusions.

\section{Financial \& competing interests disclosure}

The author is employees of Genentech, a member of the Roche Group. The authors have no other relevant affiliations or financial involvement with any organization or entity with a financial interest in or financial conflict with the subject matter or materials discussed in the manuscript apart from those disclosed.

No writing assistance was utilized in the production of this manuscript.

\section{References}

1. Lee JW, Kelley M, King LE et al. Bioanalytical approaches to quantify "total" and "free" therapeutic antibodies and their targets: technical challenges and PK/PD applications over the course of drug development. AAPS J. 13(1), 99-110 (2011).

2. Stefanini MO, Wu FT, Mac Gabhann F, Popel AS. Increase of plasma VEGF after intravenous administration of bevacizumab is predicted by a pharmacokinetic model. Cancer Res. 70(23), 9886-9894 (2010).

3. Fischer SK, Yang J, Anand B et al. The assay design used for measurement of therapeutic antibody concentrations can affect pharmacokinetic parameters: case studies. MAbs 4(5), 623-631 (2012).

4. Kuang B, King L, Wang HF. Therapeutic monoclonal antibody concentration monitoring: free or total? Bioanalysis 2(6), 1125-1140 (2010).

5. Lowe PJ, Tannenbaum S, Gautier A, Jimenez P. Relationship between omalizumab pharmacokinetics, IgE pharmacodynamics and symptoms in patients with severe persistent allergic (IgE-mediated) asthma. Br. J. Clin. Pharmacol. 68(1), 61-76 (2009).

6. Panoilia E, Schindler E, Samantas E et al. A pharmacokinetic binding model for bevacizumab and VEGF165 in colorectal cancer patients. Cancer Chemother. Pharmacol. 75(4), 791-803 (2015).

7. Richards S, Amaravadi L, Pillutla R et al. 2016 White Paper on recent issues in bioanalysis: focus on biomarker assay validation (BAV): (Part 3 - LBA, biomarkers and immunogenicity). Bioanalysis 8(23), 2475-2496 (2016).

8. Staack RF, Jordan G, Heinrich J. Mathematical simulations for bioanalytical assay development: the (un-)necessity and (im-)possibility of free drug quantification. Bioanalysis 4(4), 381-395 (2012).

9. Schick E, Staack RF, Haak M et al. Validation of a ligand-binding assay for active protein drug quantification following the 'free analyte QC concept'. Bioanalysis 8(24), 2537-2549 (2016).

10. US Department of Health and Human Services, US FDA. Guidance for Industry - Bioanalytical Method Validation (Revised) (2013). www.fda.gov/downloads/drugs/guidances/ucm368107.pdf

11. Peng K, Xu K, Liu L et al. Critical role of bioanalytical strategies in investigation of clinical PK observations, a Phase I case study. MAbs 6(6), 1500-1508 (2014). 
